\title{
Elevated Src expression associated with hepatocellular carcinoma metastasis in northern Chinese patients
}

\author{
RAN ZHAO $^{1,2^{*}}$, YIQI WU ${ }^{1,2^{*}}$, TIANZHEN WANG $^{1}$, YUHUA ZHANG $^{1}$, DAN KONG $^{3}$, LEI ZHANG $^{1}$, \\ XIAOBO LI ${ }^{1}$, GUANGYU WANG ${ }^{4}$, YINJI JIN ${ }^{1}$, XIAOMING JIN ${ }^{1,5}$ and FENGMIN ZHANG ${ }^{2,5}$ \\ Departments of ${ }^{1}$ Pathology and ${ }^{2}$ Microbiology, Basic Medical Science College, Harbin Medical University; \\ Departments of ${ }^{3}$ Gynecology and ${ }^{4}$ Oncology, Third Affiliated Hospital of Harbin Medical University; \\ ${ }^{5}$ Heilongjiang Provincial Key Laboratory for Infection and Immunity, Harbin Medical University, \\ Harbin, Heilongjiang 150081, P.R. China
}

Received October 21, 2014; Accepted July 30, 2015

DOI: $10.3892 / 01.2015 .3706$

\begin{abstract}
Src, a non-receptor type of tyrosine, was recently reported to modulate multiple signaling pathways in human tumors. Therefore, the present study aimed to determine the expression and distribution of Src on hepatocellular carcinoma (HCC). The expressions of total Src (t-Src) and an active form of Src [phosphorylated (p-) Y416Src] were analyzed in 52 northern Chinese patients with HCC using immunohistochemistry. The positive expression rates of t-Src and p-Y416Src in HCC tissue were 65.38 and $42.30 \%$, respectively, which is significantly higher than that in adjacent non-tumor tissue (30.76 and $13.46 \%$; $\mathrm{P}<0.001$ and $\mathrm{P}=0.010$, respectively). The staining intensity of $\mathrm{t}-\mathrm{Src}$ and $\mathrm{p}$-Y416Src were also significantly higher in HCC tissues compared with adjacent normal tissues $(\mathrm{P}<0.001$ and $\mathrm{P}=0.023$, respectively). $\mathrm{t}-\mathrm{Src}$ expression was positively and significantly correlated with tumor stage $(\mathrm{P}=0.002)$, cellular differentiation $(\mathrm{P}=0.007)$, metastasis $(\mathrm{P}=0.030)$ and the expression level of CA19-9 $(\mathrm{P}=0.016)$, while $\mathrm{p}-\mathrm{Y} 416 \mathrm{Src}$ expression was only significantly correlated with tumor stage $(\mathrm{P}=0.010)$. The expression of t-Src and p-Y416Src were also investigated using immunocytochemistry in two HCC cell lines with different metastatic potentials (MHCC97-L and HCCLM3) that are derived from a single HCC patient. Consistently, the expression of t-Src and p-Y416Src were stronger in the cells with higher
\end{abstract}

Correspondence to: Professor Xiaoming Jin, Department of Pathology, Basic Medical Science College, Harbin Medical University, 157 Baojian Road, Nangang, Harbin, Heilongjiang 150081, P.R. China

E-mail: jinxm55@yahoo.com.cn

Professor Fengmin Zhang, Department of Microbiology, Basic Medical Science College, Harbin Medical University, 157 Baojian Road, Nangang, Harbin, Heilongjiang 150081, P.R. China

E-mail: fengminzhang@aliyun.com

*Contributed equally

Key words: hepatocellular carcinoma, metastasis, total Src, phosphorylated Src, expression metastatic potential compared with those exhibiting lower metastatic potential. Taken together, the current data indicate that $\mathrm{Src}$ expression is elevated and active in Chinese patients with HCC and that $\mathrm{t}-\mathrm{Src}$ may have a key role in promoting HCC metastasis.

\section{Introduction}

Hepatocellular carcinoma (HCC) is the most common type of liver cancer worldwide (1). It is the third leading cause of cancer-related mortality globally and the second leading cause in China (2,3). Worldwide, there are 560,000 new cases of HCC reported per year (4). The highest incidence rates are in areas of Asia and Africa, where individuals are at a high risk of hepatitis $\mathrm{B}$ virus (HBV) and hepatitis $\mathrm{C}$ virus (HCV) infection, and it is generally accepted that hepatitis viruses have a major role in HCC development (5). A previous study (6) reported that the five-year survival rate of HCC patients following surgical resection for small and large HCC was 63.4 and 39.6\%, respectively, whereas the five-year survival rate for patients with unresectable HCC treated with cytoreduction therapy was $64.7 \%$. The poor prognosis of HCC may be attributed to the recurrent and metastatic nature of the disease (6). Therefore, the identification of oncogenes and progression markers may contribute greatly to the prevention and treatment of HCC.

c-Src, the human homolog of the Rous sarcoma virus-transforming gene, is a non-receptor tyrosine kinase. It is a critical modulator of multiple signaling pathways mediated by integrins, $\mathrm{G}$ protein-coupled receptors, cell adhesion proteins and hormone receptors (7). Src remains inactive in the cytoplasm when it is phosphorylated at Y530. However, once activated, Src translocates to the membrane and becomes fully activated by autophosphorylation at Y416 $(8,9)$. Activated Src [phosphorylated (p-) Y416Src] at the cell membrane initiates signaling pathways that induce cell proliferation, adhesion and migration/invasion (10). Src is characterized as an oncogene, and the overexpression and/or elevated activity of Src appears to be involved in the progression of various tumor types, including HCC (11,12). Notably, although higher Src activity has been detected by an in vitro kinase assay in HCC (13) and its activation is reported to be involved in the cancerous behaviors of HCC cells $(14,15)$, it has yet to be clarified whether Src 
is involved in the pathogenesis and progression of HCC, and whether it influences specific HCC clinicopathological factors.

Therefore, the present study aimed to characterize the expression and distribution of total Src (t-Src) and p-Y416Src in HCC tissues, and in two HCC cell lines with different metastatic potentials derived from a single Chinese HCC patient. Furthermore, the associations between the expression of t-Src and p-Y416Src and various clinicopathological characteristics were analyzed.

\section{Materials and methods}

Cell lines and culture. MHCC97-L and HCCLM3 cell lines, derived from MHCC97 parental HCC cells and exhibiting different metastatic potentials, were provided by the Liver Cancer Institute, Shanghai Medical College of Fudan University (Shanghai, China). MHCC97 cells are derived from a single Chinese patient with HCC $(16,17)$. All cell lines were cultured in Dulbecco's modified Eagle's medium (DMEM; GE Healthcare Life Sciences, Logan, UT, USA), containing 10\% fetal bovine serum (Gibco Life Technologies, Carlsbad, CA, USA) and $1 \%$ antibiotic (100 IU/ml penicillin and $100 \mu \mathrm{g} / \mathrm{ml}$ streptomycin; Mediatech, Inc., Manassas, VA, USA). Cells were maintained at $37^{\circ} \mathrm{C}$ in an atmosphere of $5 \% \mathrm{CO}_{2}$.

Tissue samples. A total of 52 paraffin-embedded HCC tissue samples and 52 control tissues samples from the adjacent noraml liver were obtained from Chinese patients at The First Affiliated Hospital of Harbin Medical University (Harbin, China) between 2010 and 2012. The patients were well-characterized for clinical, pathological and phenotypic markers. Sample collection was approved by the Harbin Medical University Institutional Ethics Committee and written informed consent was obtained from all patients. Long-term follow-up data was not available as all HCC cases were recent; therefore, survival curves could not be calculated. The diagnoses of HCC were established by clinical features, according to the National Comprehensive Cancer Network (18) and British Society of Gastroenterology guidelines (19), and confirmed by histological analysis. Liver specimens were obtained by needle biopsy or surgical resection and transferred to $10 \%$ neutral formalin within $15 \mathrm{~min}$ to minimize loss of phospho-antigens. All patient features, including age, tumor size, tumor node metastasis (TNM) stage (20), HBV status and $\alpha$-fetoprotein (AFP) levels, were obtained from the pathological case reports.

Immunohistochemistry (IHC). Formalin-fixed paraffin-embedded liver sections ( $5 \mu \mathrm{m}$ thick) were prepared. $\mathrm{t}-\mathrm{Src}$ and activated p-Y416Src expression were assessed by IHC using rabbit anti-human antibodies against t-Src (monoclonal IgG; 36D10; \#2109; Cell Signaling Technology, Inc., Danvers, MA, USA) and p-Y416Src (polyclonal; \#2101; Cell Signaling Technology, Inc.). The standard indirect IHC method was performed, as previously described in our laboratory (21). Briefly, the sections were placed in citrate buffer $(\mathrm{pH} \mathrm{6.0)}$ and heated in a microwave oven for antigen retrieval $\left(95^{\circ} \mathrm{C}\right.$ for $3 \mathrm{~min})$. Endogenous peroxidase activity was inhibited by incubation in $3 \%$ hydrogen peroxide $\left(\mathrm{H}_{2} \mathrm{O}_{2}\right)$ for $15 \mathrm{~min}$. After blocking with $10 \%$ goat serum for $1 \mathrm{~h}$, slides were incubated overnight at $4^{\circ} \mathrm{C}$ with antibodies to t-Src (1:400 dilution) and
Table I. Demographics, pathological features and clinical markers in the patients with hepatocellular carcinoma.

\begin{tabular}{|c|c|}
\hline Characteristic & Value \\
\hline Patients, $\mathrm{n}$ & 52 \\
\hline \multicolumn{2}{|l|}{ Gender, $\mathrm{n}$} \\
\hline Male & 42 \\
\hline Female & 10 \\
\hline \multicolumn{2}{|l|}{ Age, years } \\
\hline Mean & 54 \\
\hline Range & $22-72$ \\
\hline HBV-positive, $\%$ & 65.38 \\
\hline HCV-positive, $\%$ & 9.62 \\
\hline Cirrhosis-positive, $\%$ & 51.92 \\
\hline \multicolumn{2}{|l|}{ TNM stage, $\mathrm{n}$} \\
\hline $\mathrm{I}$ & 0 \\
\hline II & 20 \\
\hline III & 25 \\
\hline IV & 7 \\
\hline \multicolumn{2}{|l|}{ Differentiation, $\mathrm{n}$} \\
\hline Well & 13 \\
\hline Moderate & 28 \\
\hline Poor & 11 \\
\hline Lymph node metastasis-positive, $\%$ & 42.31 \\
\hline \multicolumn{2}{|l|}{ Marker expression } \\
\hline AFP $>400 \mathrm{ng} / \mathrm{ml}, \%$ & 73.08 \\
\hline $\mathrm{CEA}>5 \mu \mathrm{g} / 1, \%$ & 30.77 \\
\hline $\mathrm{SF}>13 \mu \mathrm{g} / 1, \%$ & 75.00 \\
\hline CA19-9>35 U/ml, \% & 32.69 \\
\hline
\end{tabular}

$\mathrm{HBV}$, hepatitis B virus; $\mathrm{HCV}$, hepatitis $\mathrm{C}$ virus; TNM, tumor node metastasis; AFP, $\alpha$-fetoprotein; CEA, carcinoembryonic antigen; SF, serum ferritin; CA19-9, carbohydrate antigen 19-9.

p-Y416Src (1:50 dilution). The secondary antibody staining kit (horseradish peroxidase-conjugated goat anti-rabbit IgG polymer; PV-6001; ZSGB-Bio, Beijing, China) was then applied for $45 \mathrm{~min}$ at $37^{\circ} \mathrm{C}$, and a 3,3'-diaminobenzidine substrate kit (ZLI-9019; ZSGB-Bio) was added to the tissue for $2 \mathrm{~min}$. Sections were counterstained with hematoxylin (Sigma-Aldrich, St.Louis, MO,USA), dehydrated and mounted. The specificity of immunostaining was evaluated by replacing the primary t-Src and p-Y416Src antibodies with non-specific, isotype-matched rabbit IgG (24E10; \#3195; 1:200 dilution; Cell Signaling Technology, Inc.) in phosphate-buffered saline.

Scoring. Src expression in tumor samples was assessed using the histoscore method developed by Allred et al (22). Cellular location (cytoplasm and membranes) of Src staining was scored separately for each sample. In each specimen, an intensity score and a proportion score were determined. The staining intensity was scored based on visual assessment of brown color within the cytoplasm or cell membrane on a scale of $0-3$, as follows: 0 , no staining; 1 , weak staining; 2 , moderate 
Table II. Expression of t-Src and p-Y416Src in HCC tissue and adjacent normal liver tissue $(\mathrm{n}=52)$.

\begin{tabular}{lllr}
\hline Variable & HCC, \% (n) & Normal \% (n) & $\chi^{2}$ \\
\hline t-Src positive & $65.38(34 / 52)$ & $30.76(16 / 52)$ & 12.48 \\
p-Y416Src positive & $42.30(22 / 52)$ & $13.46(7 / 52)$ & 10.76 \\
\hline
\end{tabular}

The $\chi^{2}$ test was used to analyze the frequency of Src expression. t-Src, total src; p-Y416Src, phosphorylated-Y416Src; HCC, hepatocellular carcinoma.

Table III. Subcellular localization of t-Src and p-Y416Src in HCC tissue and adjacent normal liver tissue (n=52).

\begin{tabular}{|c|c|c|c|c|}
\hline Variable & $\mathrm{HCC}, \%(\mathrm{n})$ & Normal, \% (n) & $\chi^{2}$ & P-value \\
\hline \multicolumn{5}{|l|}{$\mathrm{t}-\mathrm{Src}$} \\
\hline Cytoplasm & $65.38(34 / 52)$ & $30.76(16 / 52)$ & 12.48 & $<0.001$ \\
\hline Membrane & $21.15(11 / 52)$ & $0.00(0 / 0)$ & 12.30 & $<0.001$ \\
\hline \multicolumn{5}{|l|}{ p-Y416Src } \\
\hline Cytoplasm & $9.62(5 / 52)$ & $13.46(7 / 52)$ & 0.38 & 0.539 \\
\hline Membrane & $32.69(17 / 52)$ & $0.00(0 / 0)$ & 20.32 & $<0.001$ \\
\hline
\end{tabular}

The $\chi^{2}$ test was used to analyze the frequency of Src expression. t-Src, total src; p-Y416Src, phosphorylated-Y416Src; HCC, hepatocellular carcinoma.

staining; or 3, strong staining). The proportion score represented the percentage of positively stained cells in the entire tissue section under microscopic observation (Eclipse E800; Nikon Corporation, Tokyo, Japan) observation 0, none; $1,<5 \% ; 2,5-25 \%$; 3, 26-50\%; 4, 51-75\%; 5, >75\%). Overall Src expression in each tumor sample was then calculated as a sum of the intensity score (0-3) and the proportion score (0-5) to give a range of 0-8 (22). Scores of 0 were categorized as negative staining and scores of $1-8$ were categorized as positive staining (weak, 1-2; moderate, 3-6; positive, 7-8). Three investigators, blinded to the patient characteristics, scored the slides independently and an agreement was reached for all samples.

Immunocytochemistry. MHCC97-L and HCCLM-3 cells were cultured and fixed in 95\% ethanol (Tianjin Ke Mi Ou Chemical Reagent Co., Tianjin, China) for $5 \mathrm{~min}$ at room temperature. Cell slides were then washed and permeabilized by incubation with $0.2 \%$ Triton X-100 (Sigma-Aldrich) for $15 \mathrm{~min}$ at $4^{\circ} \mathrm{C}$. Endogenous peroxidase activity was inhibited by incubation of the cells in $3 \% \mathrm{H}_{2} \mathrm{O}_{2}$ for $20 \mathrm{~min}$. Staining of the cells using the standard indirect horseradish peroxidase method was performed as described for IHC.

Statistical analysis. Statistical analyses were performed using SPSS software (version 10.0; SPSS, Inc., Chicago, IL, USA). The $\chi^{2}$ test was used to analyze the frequency of expression, activation and subcellular localization of Src between cancer and normal tissue samples, and between different groups of clinical data. The histoscore values were reported as the mean \pm standard error of the mean. The Mann-Whitney $\mathrm{U}$ test was used to analyze differences in the expression of $\mathrm{t}-\mathrm{Src}$ and $\mathrm{p}-\mathrm{Y} 416 \mathrm{Src}$ in the cytoplasm and membrane between cancer tissue and normal tissue, and between lymph nodes with different metastatic statuses. $\mathrm{P}<0.05$ was considered to indicate a statistically significant difference.

\section{Results}

Patient characteristics. The clinical features and pathological findings of the patients investigated are indicated in Table I. Age, gender distribution, percentage of HBV- and HCV-positive patients, pathological characteristics and blood marker expression were similar to previous results in Chinese patients with HCC (23).

Expression pattern of Src in HCC and adjacent normal liver tissues. To identify the expression pattern of Src in HCC and adjacent normal liver tissues, the present study initially detected and compared the expression rate of Src in two groups of tissues. The t-Src positive expression rate in HCC tissues was $65.38 \%$, which was significantly higher than that in adjacent normal liver tissue $(30.76 \%$; $\mathrm{P}<0.001$; Table II). Similarly, the active Src (p-Y416Src) positive frequency was significantly higher in HCC tissue when compared with adjacent normal liver tissue $(\mathrm{P}=0.010$; Table II). In addition, the histoscore of t-Src and p-Y416Src staining were analyzed using a Mann-Whitney U test. The statistical analysis revealed that the staining scores of t-Src (mean histoscore, 3.21) and p-Y416Src (mean histoscore, 0.94) were both significantly higher in HCC tissues compared with in adjacent normal liver tissues $(\mathrm{P}<0.001$ and $\mathrm{P}=0.023$, respectively; Fig. 1A).

Subsequently, the subcellular distribution of Src was detected in the two groups of tissues. Of the HCC tissue samples, 34 exhibited positive t-Src staining in the cytoplasm, with different histoscores. Positive t-Src staining in the membrane was observed in 11 of the t-Src cytoplasm positive cases 


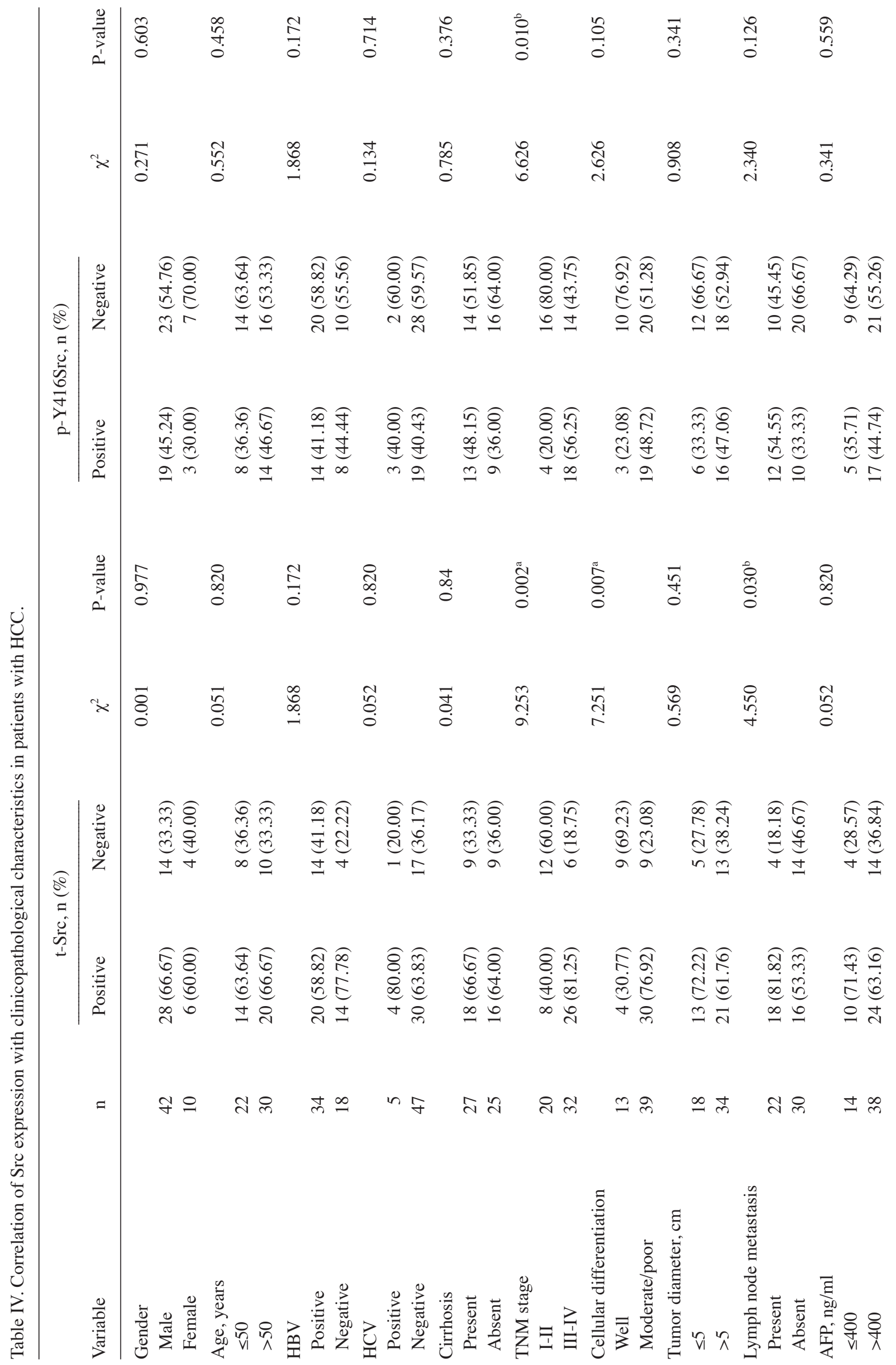


(Table III and Fig. 2A). Furthermore, positive t-Src staining in the membrane only appeared in cases of $\mathrm{HCC}$ with strong cytoplasmic staining; no cases exhibited only t-Src membrane staining. In comparison, positive t-Src staining exhibited only weak cytoplasmic distribution in hepatocytes of the adjacent normal tissue samples (Fig. 2E), whilst membrane t-Src was not detected in the normal hepatocytes (Table III). The positive staining frequency and histoscores of t-Src subcellular location in the cytoplasm (mean histoscore, 3.21; $\mathrm{P}<0.001$ and $\mathrm{P}<0.001$, respectively) and membrane (mean histoscore, 1.44; $\mathrm{P}<0.001$ and $\mathrm{P}<0.001$, respectively) were significantly higher in HCC tissues when compared with adjacent normal tissues (mean cytoplasm and membrane histoscores, 0.67 and 0.00, respectively; Table III and Fig. 1B).

With regard to $\mathrm{p}-\mathrm{Y} 416 \mathrm{Src}$, positive cytoplasmic staining was identified in $9.62 \%(5 / 52)$ of HCC cases and positive staining for membrane p-Y416Src was observed in 32.69\% (17/52) of HCC cases (Table III and Fig. 2C). By contrast, all p-Y416Src-positive adjacent normal tissue cases $(n=7)$ exhibited only weak cytoplasmic staining (Fig. 2F) and no positive staining of the membrane was detected. Statistical analysis revealed that membrane $\mathrm{p}-\mathrm{Y} 416 \mathrm{Src}$ expression was detected significantly more frequently $(\mathrm{P}<0.001)$ and more strongly $(\mathrm{P}<0.001)$ in HCC tissue (mean histoscore, 0.75$)$ than in normal liver tissue (mean histoscore, 0.00 ; Table III and Fig. 1C). However, there was no significant difference in cytoplasmic p-Y416Src staining between HCC and normal tissues (Table III and Fig. 1C).

Clinical and pathological correlations. As t-Src expression occurred at a high frequency in HCC tissues compared with adjacent normal tissues, the correlation between t-Src expression and the patients' clinicopathological characteristics was analyzed. t-Src expression was not associated with patient age, gender, HBV/HCV infection status, cirrhosis, tumor size, or AFP, carcinoembryonic antigen or serum ferritin levels $(\mathrm{P}>0.05)$. However, high expression of $\mathrm{t}-\mathrm{Src}$ in $\mathrm{HCC}$ was significantly associated with more advanced TNM cancer stage $(\mathrm{P}=0.002)$, poor cellular differentiation $(\mathrm{P}=0.007)$, the presence of lymph node metastasis $(\mathrm{P}=0.030)$ and high carbohydrate antigen 19-9 (CA19-9) level ( $\mathrm{P}=0.016$; Table IV). However, positive $\mathrm{p}-\mathrm{Y} 416 \mathrm{Src}$ expression was only significantly associated with more advanced TNM stage $(\mathrm{P}=0.010)$, and no other factors. Furthermore, strong staining of t-Src and p-Y416Src was observed in lymph nodes with HCC metastasis (Fig. 2G and H), and high Src expression scores in HCC tissues were associated with metastasis-positive lymph nodes $(\mathrm{P}=0.007, \mathrm{P}=0.008$; Table $\mathrm{V})$. These data indicated that elevated $\mathrm{t}$-Src expression was significantly associated with HCC metastasis, as well as tumor stage, cellular differentiation and CA19-9 level.

Src expression in HCC cell lines. Additionally, the expression of t-Src and p-Y416Src was detected by immunocytochemistry in two HCC cell lines with different metastatic potentials derived from a single Chinese HCC patient. Stonger expression of both t-Src and p-Y416Src was detected in the higher metastatic potential cell line, HCCLM-3 (Fig. 3B and D). However, only weak expression of these two forms of Src was detected in the lower metastatic potential cell line, MHCC97-L (Fig. 3A 
Table V. Association between lymph node status and Src expression in HCC tissues.

\begin{tabular}{lccc}
\hline Variable & $\begin{array}{c}\text { Lymph node metastasis } \\
\text { positive, } \%(\mathrm{n}=22)\end{array}$ & $\begin{array}{c}\text { Lymph node metastasis } \\
\text { negative, \% }(\mathrm{n}=30)\end{array}$ & 0.008 \\
\hline t-Src & $4.45 \pm 2.91$ & $2.30 \pm 2.62$ & 0.007 \\
p-Y416Src & $1.36 \pm 1.09$ & $0.53 \pm 1.00$ & 0.09 \\
\hline
\end{tabular}

Mean histoscore values \pm standard error of the mean were calculated for t-Src and p-Y416Src expression in lymph node with or without HCC metastasis. HCC, hepatocellular carcinoma; t-Src, total Src; p-Y416Src, phosphorylated-Y416Src.

A

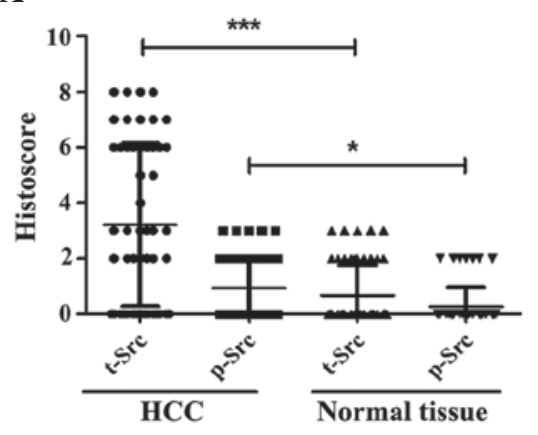

B

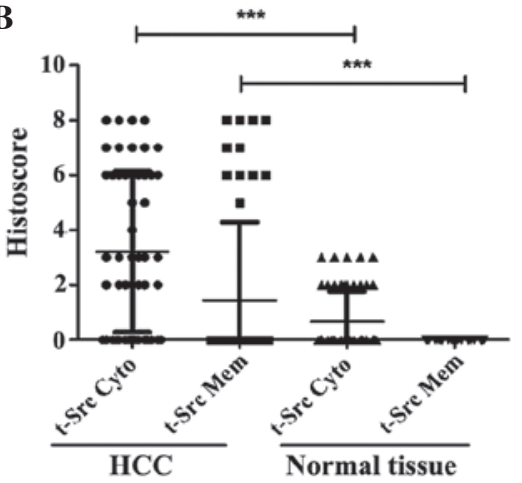

C

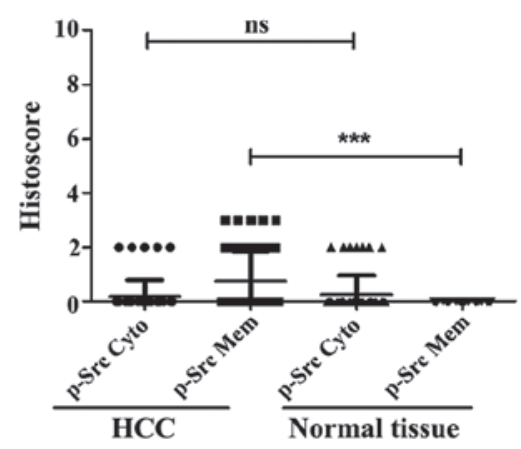

Figure 1. Expression and subcellular location of t-Src and p-Y416Src in HCC tissues and adjacent normal liver tissues. (A) Comparison of t-Src and p-Y416Src histoscores between HCC tissue and normal liver tissue. (B) Comparison of t-Src subcellular localization in the cytoplasm and membrane between HCC tissue and normal liver tissue. (C) Comparison of p-Y416Src expression in the cytoplasm and membrane of $\mathrm{HCC}$ tissue and normal liver tissue. ${ }^{*} \mathrm{P}<0.05$; ${ }^{* *} \mathrm{P}<0.01$; ${ }^{* * *} \mathrm{P}<0.001$; ns, not significant. HCC, hepatocellular carcinoma; t-Src, total Src; p-Src, phosphorylated-Src; Cyto, cytoplasm; Mem, membrane.

and C). These results were consistent with those obtained from HCC tissues, which indicated that elevated Src expression is associated with HCC metastasis.

\section{Discussion}

Src and focal adhesion kinase (FAK) are two components of non-receptor intracellular tyrosine kinases that are linked by integrin-extracellular matrix interactions (9). The Src and FAK proteins function as a complex in cellular signaling networks and control numerous important biological processes within the cell (9). Although the aberrant expression and activity of Src is well documented in colon and breast cancer $(12,24,25)$, immunohistological data regarding Src expression in HCC and the association between Src expression and HCC metastasis is still lacking.

To the best of our knowledge, the current study is the first to demonstrate increased Src expression in HCC tissue from Chinese patients compared with adjacent normal liver tissue. It was identified that $65.38 \%$ (34/52) of HCC samples from Chinese patients had positive t-Src expression, as detected by IHC. Furthermore, the localization of t-Src was cytoplasmic, consistent with previous reports $(23,26)$. Furthermore, considering that the patients in the aforementioned Japanese study of HCC (26) were predominantly positive for HCV infection, while the Chinese patients in the current study were predominantly positive for HBV infection, we propose that positive t-Src expression is not induced by a specific viral infection. Ito et al (26) identified p-Y416Src expression using IHC on liver sections from 87 Japanese patients with HCC and detected active Src in $46 \%$ of the cohort. Similarly, the present study observed that $42.30 \%$ of Chinese HCC cases exhibited positive $\mathrm{p}-\mathrm{Y} 416 \mathrm{Src}$ expression. By contrast, $\mathrm{p}$-Y416Src was not detected in normal liver samples in a previous report (23) but presented weak cytoplasmic localization in the present study. Considering that Src functions in normal and tumor cells, and its activity is dependent on phosphorylation sites, we propose that Src, including p-Y416Src, is expressed in normal hepatocytes at low levels of activation. In the current study, 16 of 34 cases with t-Src and 7 of 22 cases with p-Y416Src positive expression in HCC tissues exhibited weak cytoplasmic localization in normal hepatocytes.

The clinical and pathological implications of Src expression and HCC remain to be clarified. Masaki et al (13) identified higher Src kinase activity in a small cohort of poorly differentiated HCC cases compared with normal liver tissue. Higher expression of Src was more common in well or moderately differentiated carcinomas than in poorly differentiated ones (13), contradicting data that it is typically associated with more advanced cancer. It has also been reported that Src expression is correlated with Ki-67 expression, intrahepatic metastasis, TNM stage, tumor grade and AFP expression $(23,26,27)$. The current data demonstrates that increased t-Src expression is significantly associated with more advanced TNM cancer stage, poor cellular differentiation, the presence of lymph node metastasis and high CA19-9 expression levels. These data are partly consistent with previous studies $(23,26,27)$. It is well-known that the phosphorylation of Y416 in the activation of the kinase domain upregulates the enzyme activity of $\operatorname{Src}(28,29)$, which is 


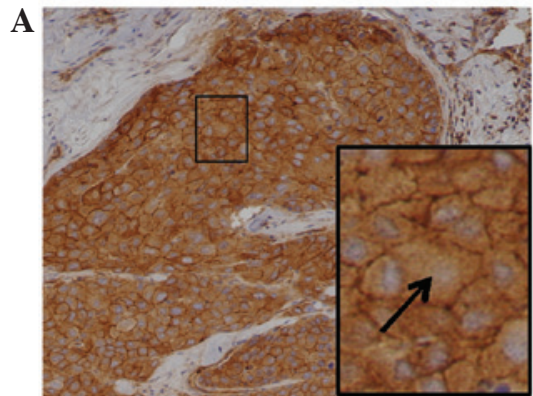

D

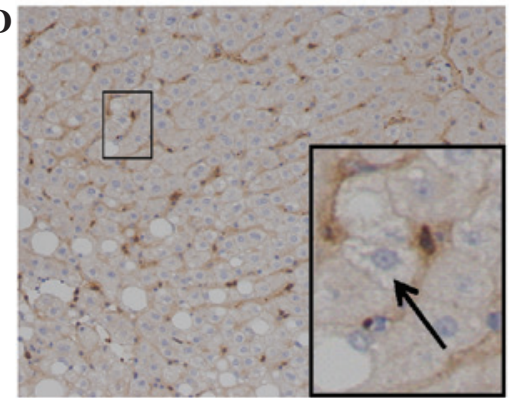

G

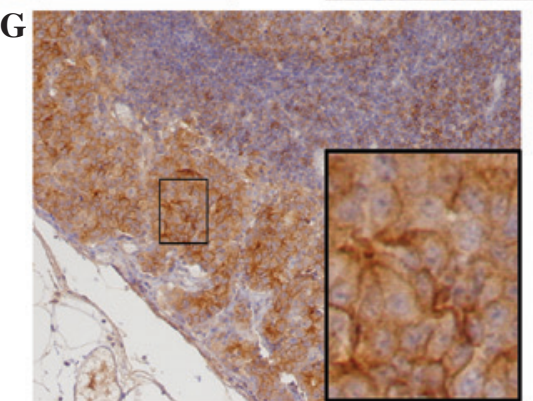

B

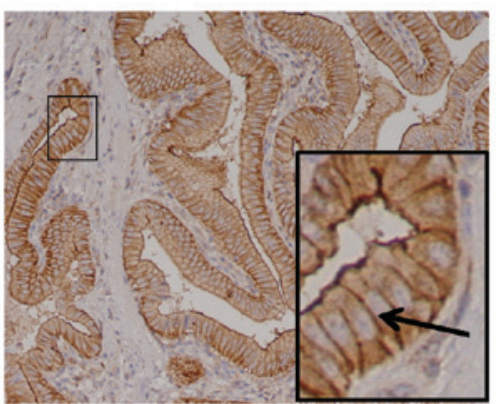

$\mathbf{E}$

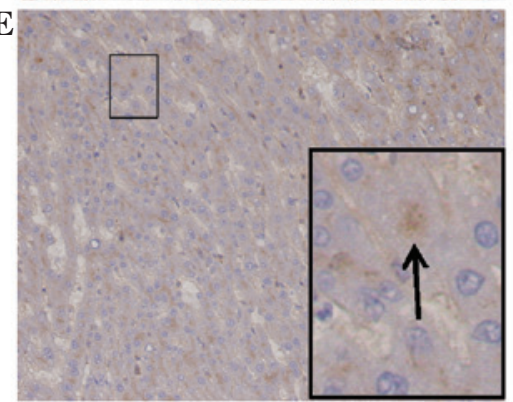

C

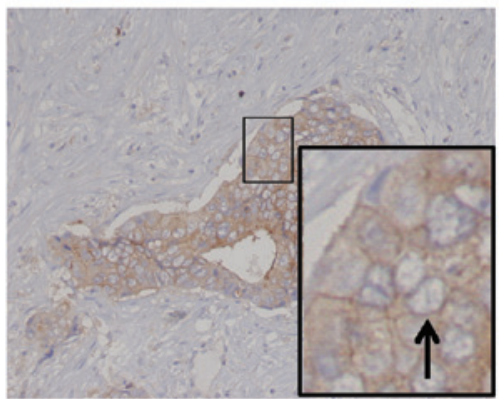

$\mathbf{F}$

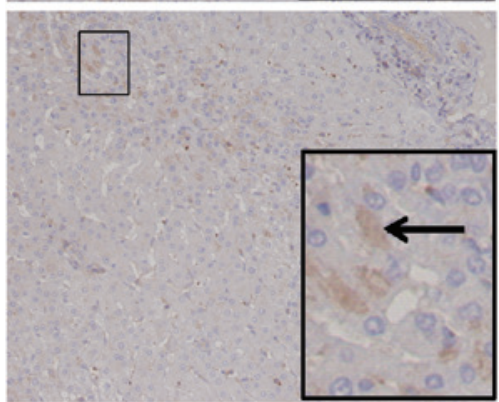

H

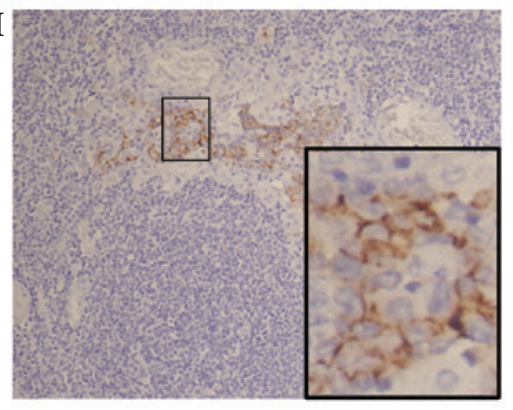

Figure 2. Src expression in HCC and adjacent normal liver tissue. Representative photomicrographs of (A) strong cytoplasmic and obvious membrane t-Src staining (arrow); (B) strong membrane t-Src staining (arrow); and (C) weak membrane p-Y416Src staining (arrow) in HCC tissue. Representative photomicrographs of (D) negative t-Src staining (arrow); (E) weak cytoplasmic t-Src staining (arrow); and (F) weak cytoplasmic p-Y416Src staining (arrow) in normal liver tissue. Representative photomicrographs of $(\mathrm{G})$ strong t-Src staining and $(\mathrm{H})$ obvious p-Y416Src membrane staining in lymph nodes with HCC metastasis (3,3'-diaminobenzidine and hematoxylin staining; magnification, x200; inset images, x16). HCC, hepatocellular carcinoma; t-Src, total Src; p-Y416Src, phosphorylated Y416Src.

A

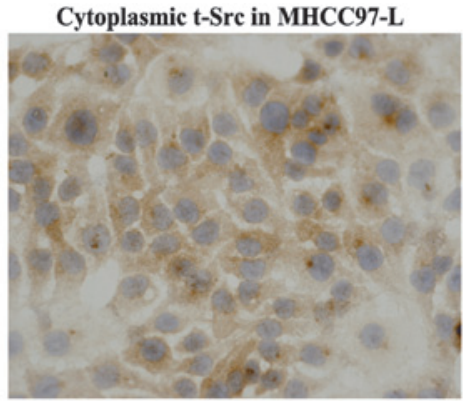

C

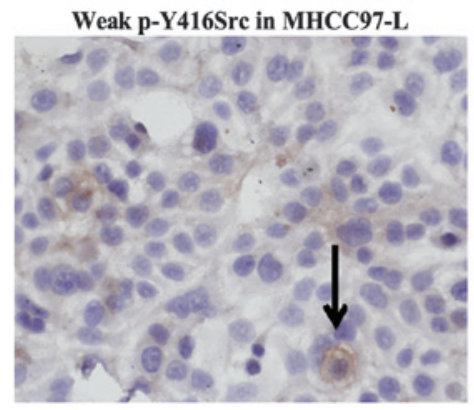

B

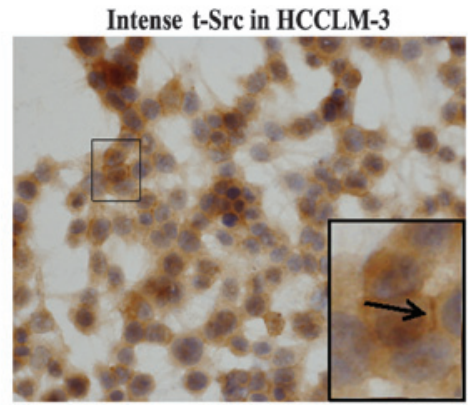

D

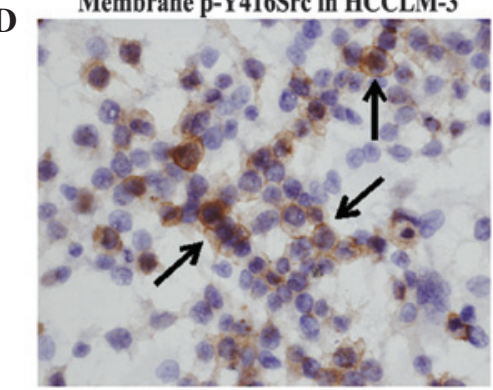

Figure 3. Src expression in two hepatocellular carcinoma cell lines. (A) Moderate cytoplasmic t-Src staining in MHCC97-L cells. (B) Cytoplasmic and membrane t-Src staining (arrow) in HCCLM-3 cells. (C) Weak p-Y416Src staining in MHCC97-L cells (arrow). (D) Membrane p-Y416Src staining in HCCLM-3 cells (arrow) (3,3'-diaminobenzidine and hematoxylin staining; magnification, x400; inset images, $\mathrm{x}$ ). t-Src, total Src; p-Y416Src, phosphorylated-Y416Src. 
involved in the induction of pathways related to cell proliferation, adhesion and migration/invasion (10). Thus, increased p-Y416Src expression may facilitate further HCC progression and is consequently associated with poor prognosis. Although it has been reported that increased p-Y416Src expression is associated with poor patient survival (27), the current data demonstrated that elevated $\mathrm{p}-\mathrm{Y} 416 \mathrm{Src}$ expression is independent of the majority of clinical and pathological parameters.

The MHCC97 cell line, a human HCC cell line with high metastatic potential, was established by Tian et al (16) using a subcutaneous xenograft of a metastatic model of human HCC from a Chinese patient in nude mice. Based on MHCC97 as the parental cells, three cell lines (MHCC97-L, MHCC97-H and HCCLM3) were subsequently established with increasing metastatic potential (17). In the present study, to support the findings of increased t-Src expression in HCC tissue, the expression of Src was detected in MHCC97-L and HCCLM-3 cell lines using immunocytochemistry. Notably, Src was more highly expressed in the cell line with the highest metastatic potential, HCCLM-3, compared with that in MHCC97-L cells (with lower metastatic potential). These data suggest that Src may be involved in HCC progression. Src overexpression or overactivation has also been identified in a variety of human biopsies from primary tumors and their metastases (11). It has been established that active Src is associated with integrin adhesion dynamics and E-cadherin dysregulation during the Src-induced epithelial-mesenchymal transition. However, the mechanisms of Src induced metastasis in $\mathrm{HCC}$ require further investigation.

In conclusion, the present study demonstrated that the expression of t-Src and p-Y416Src were markedly elevated in HCC tissue. In addition, it was observed that t-Src expression was associated with cancer stage, cellular differentiation, lymph node metastasis and CA19-9 level. Similarly to previous studies, the present results demonstrated the potential value of $\mathrm{Src}$ as predictor of HCC outcome. In addition, to the best of our knowledge, the present study is the first to identify an association between elevated t-Src and CA19-9 expression. Notably, elevated t-Src expression was observed in HCC tissues with lymph node metastasis and in an HCC cell line with a high metastatic potential. Collectively, the current data suggests that Src may be important in HCC metastasis. However, the underlying mechanisms require further investigation.

\section{Acknowledgements}

The present study was supported by the Academic Backbone Support Plan of Heilongjiang Province Department of Education (grant no. 1254G040). The authors would also like to thank the Heilongjiang Provincial Science and Technology Innovation Team in Higher Education Institutes for Infection and Immunity, and Heilongjiang Provincial Key Laboratory for Infection and Immunity, Harbin Medical University (Harbin, China) for their assistance.

\section{References}

1. Farazi PA and Depinho RA: The genetic and environmental basis of hepatocellular carcinoma. Discov Med 6: 182-186, 2006.

2. Parkin DM, Bray F, Ferlay J and Pisani P: Global cancer statistic, 2002. CA Cancer J Clin 55: 74-108, 2005.
3. He J, Gu D, Wu X, et al: Major causes of death among men and women in China. N Engl J Med 353: 1124-1134, 2005.

4. Hussain SA, Ferry DR, El-Gazzaz G, Mirza DF, James ND, McMaster P and Kerr DJ: Hepatocellular carcinoma. Ann Oncol 12: 161-172, 2001.

5. Shimizu I, Kohno N, Tamaki K, Shono M, Huang HW, He JH and Yao DF: Female hepatology: Favorable role of estrogen in chronic liver disease with hepatitis B virus infection. World J Gastroenterol 13: 4295-4305, 2007.

6. Tang Z, Zhou X, Lin Z, Yang B, Ma Z, Ye S, Wu Z, Fan J, Liu Y, Liu K, et al: Surgical treatment of hepatocellular carcinoma and related basic research with special reference to recurrence and metastasis. Chin Med J (Engl) 112: 887-891, 1999.

7. Collett MS and Erikson RL: Protein kinase activity associated with the avian sarcoma virus src gene product. Proc Natl Acad Sci USA 75: 2021-2024, 1978.

8. Ingley E: Src family kinases: Regulation of their activities, levels and identification of new pathways. Biochim Biophys Acta: 56-65, 1784, 2008

9. Bolos V, Gasent JM, Lopez-Tarruella S and Grande E: The dual kinase complex FAK-Src as a promising therapeutic target in cancer. Onco Targets Ther 3: 83-97, 2010.

10. Frame MC: Src in cancer: Deregulation and consequences for cell behaviour. Biochim Biophys Acta 1602: 114-130, 2002.

11. Yeatman TJ: A renaissance for SRC. Nat Rev Cancer 4: 470-480, 2004.

12. Chen J: Is Src the key to understanding metastasis and developing new treatments for colon cancer? Nat Clin Pract Gastroenterol Hepatol 5: 306-307, 2008.

13. Masaki T, Okada M, Shiratori Y, Rengifo W, Matsumoto K, Maeda S, Kato N, Kanai F, Komatsu Y, Nishioka M and Omata M: pp60c-src activation in hepatocellular carcinoma of human and LEC rats. Hepatology 27: 1257-1264, 1998.

14. Sun CK, Man K, Ng KT, Ho JW, Lim ZX, Cheng O, Lo CM, Poon RT and Fan ST: Proline-rich tyrosine kinase 2 (Pyk2) promotes proliferation and invasiveness of hepatocellular carcinoma cells through c-Src/ERK activation. Carcinogenesis 29: 2096-2105, 2008.

15. De Toni EN, Thieme SE, Herbst A, Behrens A, Stieber A, Jung A, Blum H, Göke $\mathrm{H}$ and Kolligs FT: OPG is regulated by beta-catenin and mediates resistance to TRAIL-induced apoptosis in colon cancer. Clin Cancer Res 14: 4713-4718, 2008.

16. Tian J, Tang ZY, Ye SL, Liu YK, Lin ZY, Chen J and Xue Q: New human hepatocellular carcinoma (HCC) cell line with highly metastatic potential (MHCC97) and its expressions of the factors associated with metastasis. Br J Cancer 81: 814-821, 1999 .

17. Li Y, Tian B, Yang J, Zhao L, Wu X, Ye SL, Liu YK and Tang ZY: Stepwise metastatic human hepatocellular carcinoma cell line model system with multiple metastatic potentials establish through consecutive in vivo selection and studies on metastatic characteristics. J Cancer Res Clin Oncol 130: 460-468, 2004.

18. National Comprehensive Cancer Network. NCCN Clinical Practice Guidelines in Oncology: Hepatobiliary cancers. Version 2.2012. http://www.nccn.org/professionals/physician_ gls/pdf/hepatobiliary.pdf. Accessed August 27, 2015.

19. Ryder SD; British Society of Gastroenterology: Guidelines for the diagnosis and treatment of hepatocellular carcinoma (HCC) in adults. Gut 52 (Suppl 3): iiil-iii8, 2003.

20. Greene FL, Page DL, Fleming ID, Fritz A, Balch CM and. Haller DG (eds): Liver Staging Form. In: AJCC Cancer Staging Manual. 6th edition. Springer-Verlag, New York, NY, p131, 2002.

21. Wu Y, Wang T, Ye S, Zhao R, Bai X, Wu Y, Abe K and Jin X: Detection of hepatitis B virus DNA in paraffin-embedded intrahepatic and extrahepatic cholangiocarcinoma tissue in the northern Chinese population. Hum Pathol 43: 56-61, 2012.

22. Allred DC, Clark GM, Elledge R, Fuqua SA, Brown RW, Chamness GC, Osborne CK and McGuire WL: Association of p53 protein expression with tumor cell proliferation rate and clinical outcome in node-negative breast cancer. J Natl Cancer Inst 85: 200-206, 1993

23. Lau GM, Lau GM, Yu GL, et al: Expression of Src and FAK in hepatocellular carcinoma and the effect of Src inhibitors on hepatocellular carcinoma in vitro. Dig Dis Sci 54: $1465-1474,2009$.

24. Morgan L, Nicholson RI and Hiscox S: SRC as a therapeutic target in breast cancer. Endocr Metab Immune Disord Drug Targets 8: 273-278, 2008 . 
25. Anbalagan M, Moroz K, Ali A, Carrier L, Glodowski S and Rowan BG: Subcellular localization of total and activated Src kinase in African American and Caucasian breast cancer. PLoS One 7: e33017, 2012.

26. Ito Y, Kawakatsu H, Takeda T, Sakon M, Nagano H, Sakai T, Miyoshi E, Noda K, Tsujimoto M, Wakasa K, et al: Activation of $\mathrm{c}$-src gene product in hepatocellular carcinoma is highly correlated with the indices of early stage phenotype. J Hepatol 35: 68-73, 2001.

27. Chen ML, Chai CY, Yeh KT, Wang SN, Tsai CJ, Yeh YT and Yang SF: Crosstalk between activated and inactivated c-Src in hepatocellular carcinoma. Dis Markers 30: 325-333, 2011.
28. Hunter T: A tail of two src's: Mutatis mutandis. Cell 49: 1-4, 1987.

29. Roskoski R: Src protein-tyrosine kinase structure and regulation. Biochem Biophys Res Commun 324: 1155-1164, 2004. 